\title{
Self-driven soft imaging in liquid by means of photothermal excitation
}

\author{
P. Paoletti, ${ }^{1, a)}$ M. Basso, ${ }^{1,2}$ V. Pini, ${ }^{3}$ B. Tiribilli, ${ }^{1}$ and M. Vassalli ${ }^{4}$ \\ ${ }^{1}$ Istituto dei Sistemi Complessi, CNR, via Madonna del Piano 10, Sesto Fiorentino, Firenze I-50019, Italy \\ ${ }^{2}$ Dipartimento di Sistemi e Informatica, Universita di Firenze, via S.Marta 3, Firenze I-50139, Italy \\ ${ }^{3}$ BioNanoMechanics Lab, IMM-CNM (CSIC), Calle Isaac Newton 8 (PTM), Tres Cantos, Madrid \\ E-28760, Spain \\ ${ }^{4}$ Istituto di Biofisica, CNR, via De Marini 6, Genova I-16149, Italy
}

(Received 12 July 2011; accepted 27 October 2011; published online 8 December 2011)

\begin{abstract}
The use of a laser to induce oscillations of an atomic force microscopy cantilever provides a way to excite the dynamics of the system in a very controlled manner. This excitation scheme has been used to obtain reliable self-sustained oscillations, in air and in liquid environments, and to implement an additional control loop leading to a soft, low-interaction, working mode. The dynamics of the oscillating cantilever has been characterized, both theoretically and experimentally, and the effectiveness of the approach has been verified on a test sample. (C) 2011 American Institute of Physics. [doi:10.1063/1.3665396]
\end{abstract}

\section{INTRODUCTION}

Since its invention in $1986,{ }^{1}$ atomic force microscopy (AFM) has been unceasingly improved in many aspects and the efforts of the scientific community have led to the introduction of new and attractive working modes and technical solutions, aimed at enhancing the imaging speed, ${ }^{2}$ reducing the interaction strength with the sample, ${ }^{3}$ or measuring additional physical parameters during the scan. ${ }^{4-6}$ In recent years, the attention was pointed at exploiting the intrinsic dynamics of the probing system (the cantilever) to attain even more sophisticated imaging modes. ${ }^{7,8}$ Among them, implementations based on selfexcitation of the probe (self-driven AFM, SD-AFM) showed the ability to boost the sensitivity of the system ${ }^{9}$ and thus they were successfully applied in sensor and, in particular, biosensor applications. Nevertheless, such excitation techniques would also play a crucial role in the development of soft imaging modes required for biological applications. ${ }^{10}$ However, the implementation scheme typically adopted for SD-AFM, based on a constant-excitation circuit leading to frequencymodulation detection, ${ }^{11}$ did not prove to be sufficiently stable and reliable compared to the standard constant-amplitude approach. ${ }^{12}$ Recently, a new strategy, called auto-tapping (AT), that combines the benefits of both constant-amplitude and selfdriven excitation, has been proposed. ${ }^{13}$ The possibility of obtaining high image quality in combination with weaker-tipsample interactions has been verified in air using an additional feedback system (AT with saturation control, ATC, Ref. 14), but, to be effective for biological imaging, the validity of this approach should be verified in a liquid environment. We tested the possibility of obtaining a straightforward implementation of ATC in water but the presence of the so-called "forest of peaks" 15 in the oscillation spectrum of the cantilever induces a coupling between the modes that strongly interferes with the self-driven dynamics, often spoiling the experimental session.

To port the benefits of ATC to a liquid environment, in this work a specific optical excitation of the cantilever (opting mode) has been employed. In fact, the standard tapping

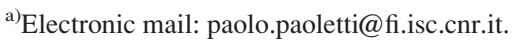

mode induces the oscillation by means of an acoustical wave generated far from the cantilever by a small dither piezo, thus eliciting several modes, not belonging to the cantilever dynamics, that couple together in the presence of a liquid medium. ${ }^{16}$ The same does not occur when using a laser directly focused on the back of the cantilever. The physics of the interaction between an amplitude modulated laser and the AFM cantilever has been quite extensively studied ${ }^{17-21}$ and it has been applied to sensing and imaging applications, in air $^{22}$ and in liquid. ${ }^{23-25}$ The advantage of using SD-AFM modes with respect to standard constant amplitude is even enhanced by using optical excitation but its implementation in water is somewhat tricky, because of the strong loss of excitation efficiency of the photo-thermal effect in a liquid environment. This drawback has been circumvented in sensing applications ${ }^{26}$ where the acquisition time is not a constraint, but the same strategy cannot be adopted for imaging applications without heavily decreasing the scan speed.

In this paper, we test the feasibility of SD-AFM with optical excitation (auto-opting, AO), and we present an implementation of the ATC algorithm, called auto-opting with saturation control (AOC), both in air and liquid environments. The experimental setup is described in Sec. II, whereas measurement results in air and in water are reported, respectively, in Secs. III and IV, with a comparison to the theoretical expectations. In Sec. V, the results are summarized and discussed.

\section{EXPERIMENTAL SETUP}

The experimental setup used for the measurements is based on a homemade $\mathrm{AFM},{ }^{20}$ with a $200 \mu \mathrm{m} \times 200 \mu \mathrm{m} \times 20 \mu \mathrm{m}$ piezo scanner (P527 by Physik Instrumente) and a detection system based on the standard optical beam deflection method (OBDM) implemented using a 635-nm red laser diode (LQB2S-635 by World Star Tech) and a four-quadrants detector (SD197-23-21-041 by Advanced Photonix). The mechanical head is mounted under an optical microscope (FS60 by Mitutoyo) useful to visualize the cantilever and to align the OBDM laser on it. The auxiliary port of the same microscope on the 
back of the cantilever is suitable to focus the excitation laser, an intensity modulated infrared diode with $880-\mathrm{nm}$ wavelength (SDL2412-7690 by Spectra Diode Labs) coupled with the system through an optical fiber.

Figure 1 presents a scheme of the whole experimental setup where an excitation laser is focused on the back of the AFM cantilever. The dashed blocks are related to the selfoscillation (AO electronics) and to the additional feedback loop (threshold controller). AO electronics is composed by a variable gain amplifier and an OPA 698 IC that performs a linear amplification of the AC component of the deflection signal and a bipolar clipping with adjustable threshold (driven by the threshold controller). The conversion of the fast oscillating signal into a stationary amplitude information is done in commercial systems by means of a lock-in amplifier tuned at the driving frequency. In SD-AFM, the system lacks a reference frequency and thus this circuit has been replaced by a fast peak detector whose output is fed to the standard PI controller, generally used to keep constant the oscillation amplitude (i.e., the tip-sample distance) during the raster scan of the sample.

The signals were acquired and conditioned using a multi-function data acquisition board (PCI 6259 by National Instruments) installed on a general purpose PC running a Linux distribution patched for real-time operations using real-time application interface (RTAI). The controller program was developed using the RTAI lab code generator tool chain based on Simulink ${ }^{\mathrm{TM}}$ and Real Time Workshop ${ }^{\mathrm{TM}}$ (Mathworks). The experiment was remotely managed via ethernet using a custom developed graphic user interface in Windows $^{\mathrm{TM}}$ 27,28 $^{27}$

\section{AO AND AOC IMAGING IN AIR}

Figure 2 shows in solid the oscillation spectrum of the cantilever using photothermal excitation in air. The experimental data have been obtained using a pretty soft cantile$\operatorname{ver}^{29}$ (CSG10 by NT-MDT) whose dynamics in AO has been theoretically studied in a previous work. ${ }^{20}$ The system shows a first resonance at $\nu \simeq 21.4 \mathrm{kHz}$, with $Q \simeq 45$ that is well fitted by the theoretical model (dashed curve), taking into account the physics of the optical excitation ${ }^{20}$ (see the calculations reported in the Appendix).

Far from the sample, the free oscillation amplitude $B_{0}$ depends linearly on the saturation threshold $s$. On the other hand, when the cantilever gets closer to the sample surface, the instantaneous oscillation amplitude $B(t)$ shows a linear relationship with the mean distance from the sample, thus it is also possible to exploit the same controller used in classical amplitude-modulation schemes for self-sustained oscillations (AO). ${ }^{13}$ Moreover, biological imaging applications require the interaction to be kept as weak as possible to avoid damaging soft samples. This goal can be achieved in classical amplitude modulation techniques by choosing the setpoint $B_{r}$ of the actuator piezo PI controller close to the free oscillation amplitude $B_{0}$. However, this in turn can worsen the image quality. In fact, if the sample topography presents a steep descending edge, the oscillating tip can jump over the step, losing contact. During this transient, the oscillation amplitude goes to $B_{0}$ and the error (the input of the feedback) attains its maximum value, $B_{0}-B_{r}$, that is a small quantity in a low-interaction setting. In this situation, the proportional gain of the feedback is almost ineffective and the recovery of the contact with the sample is entrusted only to the integral gain of the PI controller, thus leading to a slow decrease. Taking into account that the integral gain cannot be increased without destabilizing the control loop once newly in contact, the sole viable approach is reducing the scanning speed to compensate for this effect.

A possible approach to overcome this drawback has been proposed by the authors for acoustical self-excited systems. ${ }^{14}$ This solution exploits the additional degree of freedom provided by the variable threshold controller of auto-tapping to dynamically adapt the free oscillation amplitude so that the ratio $B(t) / B_{0}$ remains constant. Note that this is the same goal of the actuator piezo PI controller, but varying the saturation threshold does not involve any mechanical

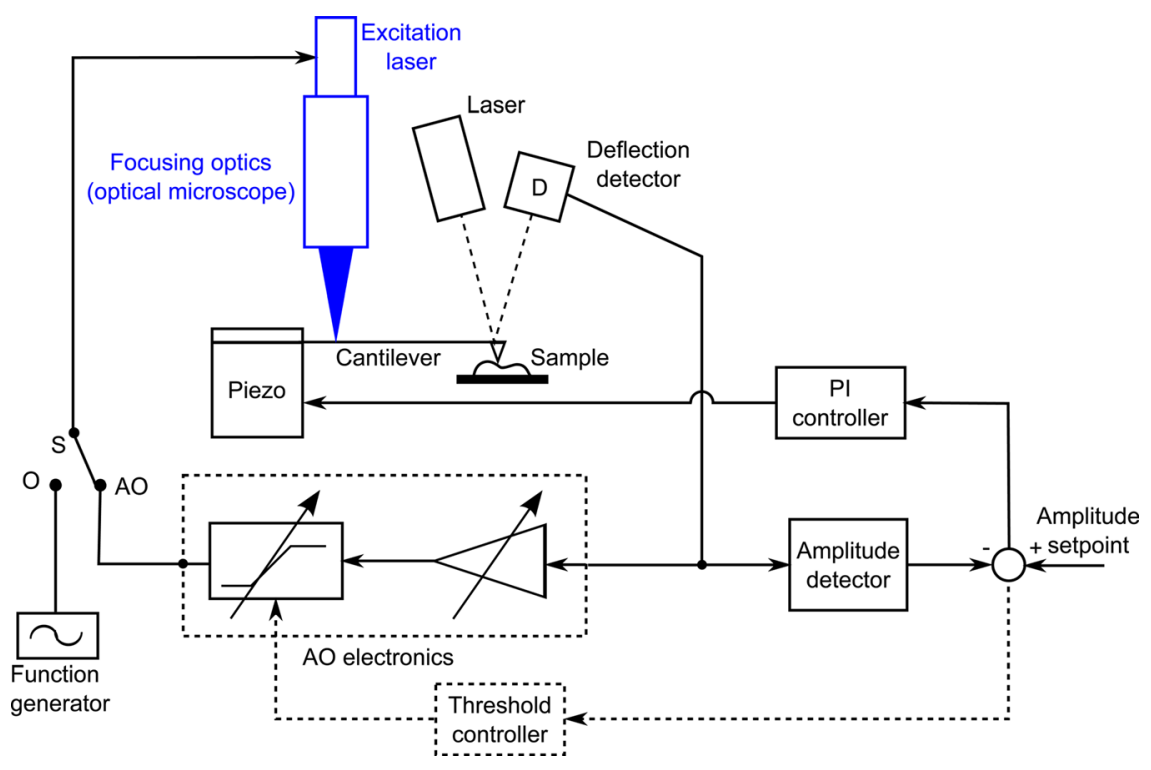

FIG. 1. (Color online) Experimental setup schematic. 


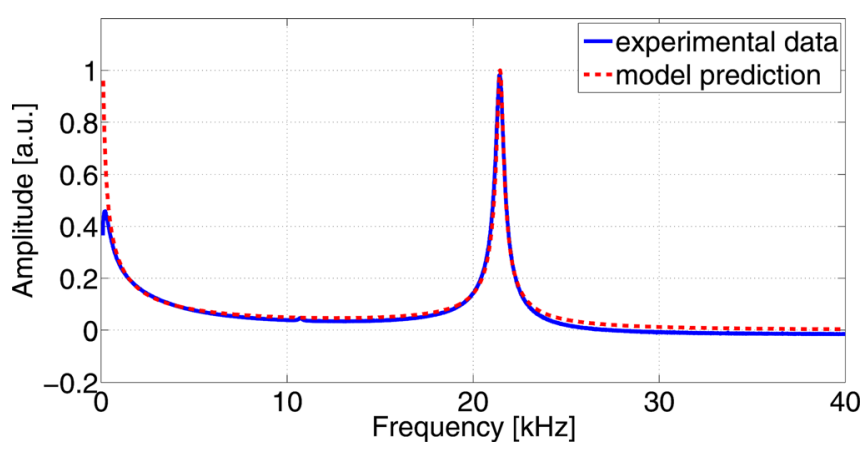

FIG. 2. (Color online) Frequency response in air of a cantilever excited in opting. Experimental data (solid line) are fitted with the theoretical expectation (dashed line)

components and therefore the bandwidth of this control action is much higher than the one achievable with piezoelectric stages. Because of this, one is then allowed to use $B_{r}$ closer to $B_{0}$ without falling into the above-mentioned phenomenon. A control law for the threshold controller is then defined as follows:

$$
s(t)=\left\{\begin{array}{ll}
s_{0}-K_{\mathrm{sat}}^{-}\left(B_{r}-B(t)\right) & B_{r}-B(t) \geq 0 \\
s_{0}-K_{\mathrm{sat}}^{+}\left(B_{r}-B(t)\right) & B_{r}-B(t)<0
\end{array},\right.
$$

where $s(t)$ represents the instantaneous saturation threshold and $s_{0}$ its default value, i.e., the one correponding to the freeoscillation amplitude $B_{0} . K_{\text {sat }}^{ \pm}$are gains that should satisfy $K_{\text {sat }}^{-}<s_{0} / B_{r} \leq K_{\text {sat }}^{+}$. Two different gains for approach and retract phases are introduced for dealing with two different control goals. ${ }^{14}$ In fact, during the approach phase $\left(B(t)<B_{r}\right)$ the controller gain $K_{\text {sat }}^{-}$must be kept small for not turning off the oscillations and, at the same time, it can be useful for reducing the mean interaction force by enforcing $B(t) / B_{0}$ close to the unitary value. On the other hand, during the retract phase $\left(B(t)>B_{r}\right)$ the oscillation amplitude should be increased as fast as possible, thus using high values for $K_{\text {sat }}^{+}$.

Figure 3 reports an image comparing the performance of $\mathrm{AO}$ and $\mathrm{AOC}$ in air. The horizontal line indicates the position at which the saturation controller is manually excluded, during the scan, switching from AOC to AO. The freeoscillation amplitude was set to about $B_{0}=40 \mathrm{~nm}$, the setpoint to $B_{r}=35 \mathrm{~nm}\left(B_{r} / B_{0}=0.875\right)$, and the scanning rate was set to 2 lines/s. The saturation threshold control law of AOC was defined by $K_{\text {sat }}^{-}=0 \mathrm{~V} / \mathrm{nm}$ and $K_{\text {sat }}^{+}=0.47 \mathrm{~V} / \mathrm{nm}$. The approaching gain $K_{\text {sat }}^{-}$was set to zero because in this case the sample stiffness is pretty high and therefore no additional benefits would be acquired by decreasing the freeoscillation amplitude. When the threshold controller is switched off, the topography of the calibration grating used for the test (TGZ01 by NT-MDT) is reconstructed with lower reliability, showing a wake on the falling edge of the grating step. This behavior is quantified in Fig. 4 where the AOC profile (solid line) is evidently faster in recovering the contact with the sample, lost at the step edge, whereas the AO profile (dashed line) shows slower dynamics, remaining out of contact for a longer time.

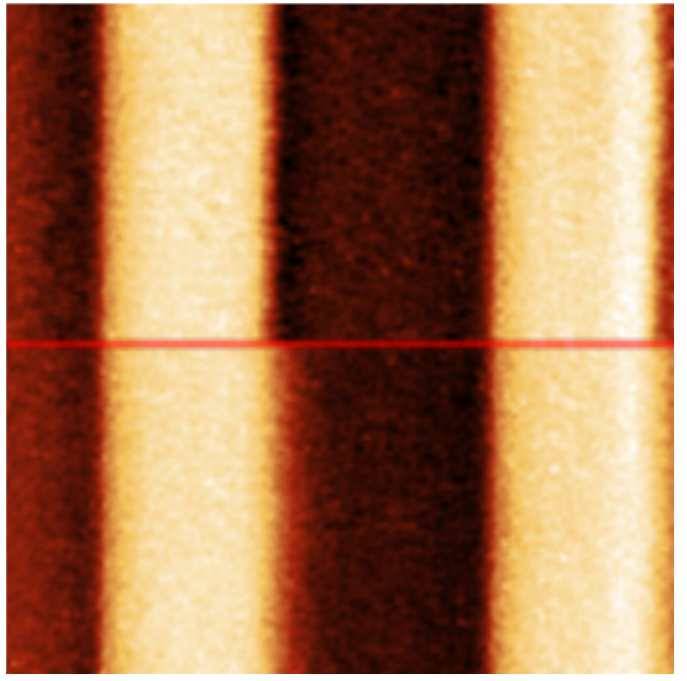

FIG. 3. (Color online) TGZ01 calibration grating image obtained in air by switching on (top) and off (bottom) the threshold controller. $5 \times 5 \mu \mathrm{m}$ $(256 \times 256$ pixels $)$ image obtained with $B_{0}=40 \mathrm{~nm}$ and set-point $B_{r}=35 \mathrm{~nm}$ at a scan speed of $12 \mu \mathrm{m} / \mathrm{s}(2 \mathrm{~Hz})$.

\section{AO AND AOC IMAGING IN LIQUID}

Switching from air to water gives rise to many issues related to the density and viscosity of the medium. It is well known that the standard tapping mode induces a coupling of the cantilever dynamics with external mechanical resonances that can be observed in the spectrum as a growing forest of peaks (see the inset in Fig. 5). The optical excitation does not suffer of this limitation because the excitation is directly applied on the cantilever and the spectrum, presented in Fig. 5, does not show any spurious effect.

The experimental results (solid line) are fitted by the theory (dashed line, see the Appendix) clearly showing the resonance peak at about $\nu=5.3 \mathrm{kHz}$, together with an increase for lower frequencies. This phenomenon, expected by the theory, is related to the characteristics of the photothermal effect whose response rises at small frequencies, depending on the parameters of the heat diffusion in the cantilever. To drive the dynamics away from this region, avoiding the establishment of non-resonant self-excitation, a high-pass filter at about $1 \mathrm{kHz}$ has been introduced in the $\mathrm{AO}$ circuit.

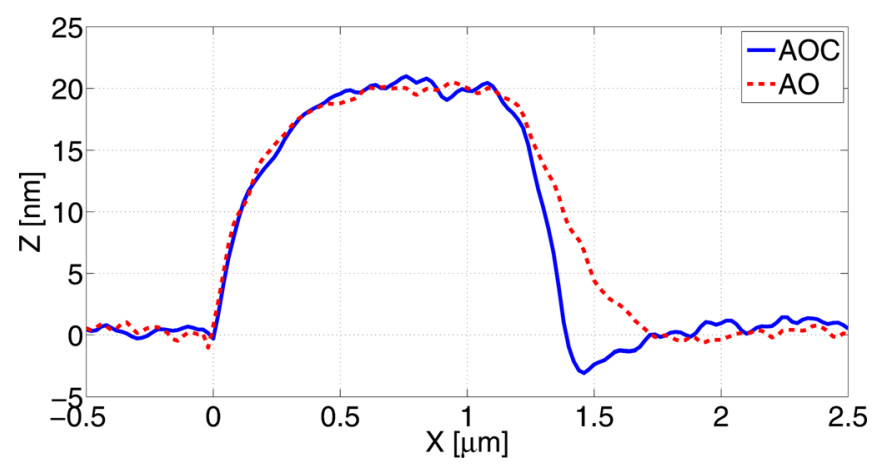

FIG. 4. (Color online) Comparison between line profiles obtained in air with (AOC, solid line) and without (AO, dashed line) saturation control. 


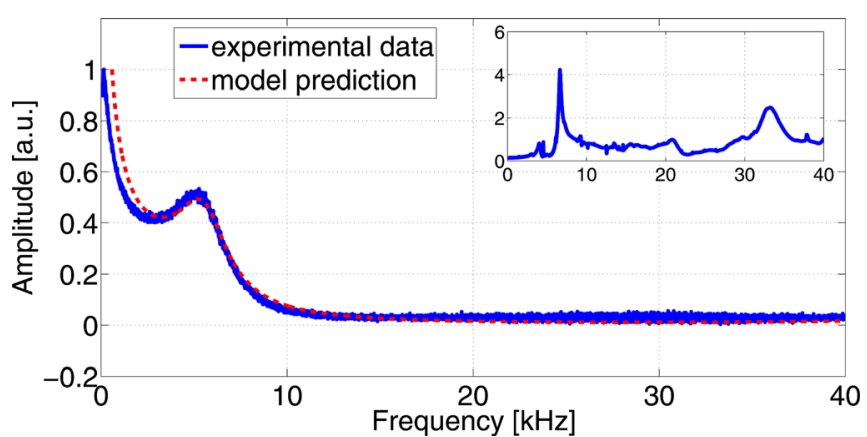

FIG. 5. (Color online) Frequency response in water of a cantilever excited in opting. Experimental data (solid line) are fitted with the theoretical expectation (dashed line). The inset shows, as a comparison, the spectrum obtained with acoustical excitation.

An experiment similar to that reported in Sec. III for the air environment has been executed to test the feasibility of AOC imaging in liquid environments. Figure 6 presents the outcome of the measurements obtained with a free oscillation amplitude of about $B_{0}=4.6 \mathrm{~nm}$ and a set-point of $B_{r}=4 \mathrm{~nm}\left(B_{r} / B_{0}=0.87\right)$. The scanning rate was set to 1 line/s and the threshold controller gains were defined as $K_{\text {sat }}^{-}=0 \mathrm{~V} / \mathrm{nm}$ and $K_{\text {sat }}^{+}=1.19 \mathrm{~V} / \mathrm{nm}$. A test image was acquired on the same TGZ01 calibration grating used for air imaging, showing an even stronger beneficial effect of the saturation controller on the topography reconstruction. This effect is better quantified in the line profile comparison reported in Fig. 7, where the settling time at the falling edge of the topography is two to three times smaller for AOC than for simple AO.

\section{FINAL REMARKS}

The realization of an optical excitation system on a custom AFM system gave the possibility to exploit the phenomenon of self-driven excitation for imaging applications in liquid. This result opens the way to the implementation of

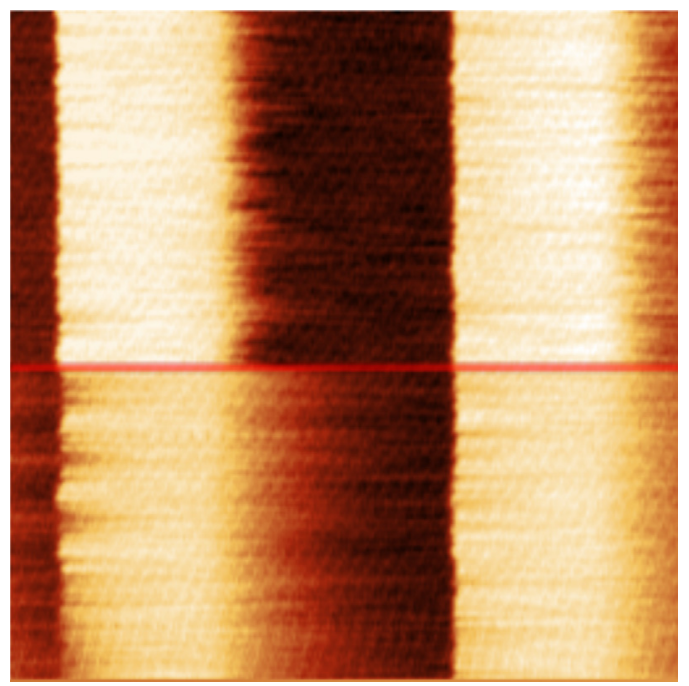

FIG. 6. (Color online) TGZ01 calibration grating image obtained in water by switching on (top) and off (bottom) the threshold controller. $5 \times 5 \mu \mathrm{m}$ $(256 \times 256$ pixels $)$ image obtained with $B_{0}=4.6 \mathrm{~nm}$ and set-point $B_{r}=4 \mathrm{~nm}$ at a scan speed of $6 \mu \mathrm{m} / \mathrm{s}(1 \mathrm{~Hz})$.

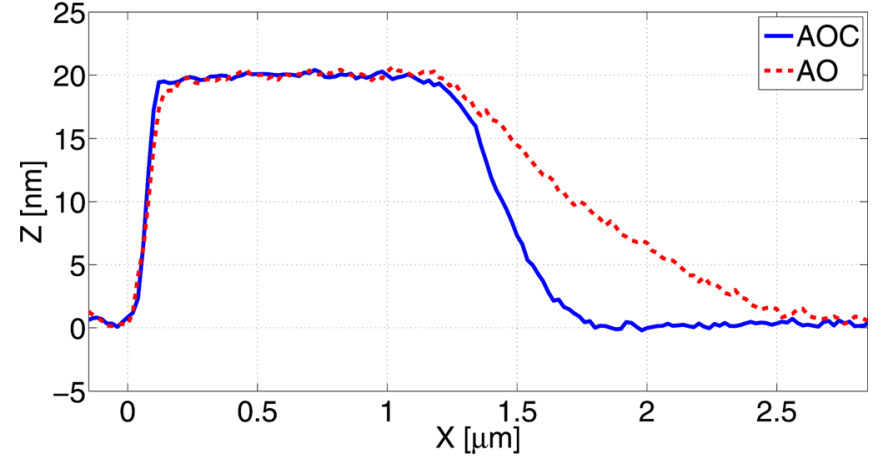

FIG. 7. (Color online) Comparison between line profiles obtained in water with (AOC, solid line) and without (AO, dashed line) saturation control.

advanced imaging modes based on self-driven dynamics. In particular, the effectiveness of the AOC saturation controller was tested, showing the possibility of imaging a sample in water with very low interaction forces. Applied to the imaging of biological samples, this "soft mode" in liquid will provide a significant enhancement, strongly reducing the probability of damaging or altering the sample.

\section{APPENDIX: THEORY OF THE PHOTOTHERMAL EFFECT ON COATED CANTILEVERS}

In this Appendix, we show a detailed calculation of the photothermal-induced force distribution $f_{\text {phot }}(x, \nu)$ used to predict the frequency response of the cantilever, both in air and in liquid. To simplify the treatment we introduce the following hypotheses:

- the force distribution is only dependent on the coordinate $x$ along the longer side of the cantilever beam;

- the spatial and temporal coordinates are separable, so that the driving force can be written as $f_{\text {phot }}(x, t)=f(x) g(t)$, i.e., we restrict the analysis to dynamical stationary conditions;

- the temporal dependence of the driving force is sinusoidal, as in our experimental setup;

- the region of the laser excitation is punctual.

The first and the latter hypotheses are justified by the fact that the laser spot size is usually comparable with the cantilever width $W$ that, in turn, is far larger than the cantilever thickness $D$ and far smaller than the cantilever length $L$. This implies that the temperature distribution is approximately uniform across the width and the thickness and, therefore, one only needs to model temperature distribution along the longer side of the cantilever. Within these settings, taking into account $W \ll L$, the laser excitation can be considered as punctual.

The leading phenomenon produced by a laser focused on a coated cantilever is the photothermal effect. The intensity-modulated light, focused onto a region of the cantilever, produces a time-dependent temperature distribution because of the absorption of optical energy that in turn induces a differential longitudinal stress across the cantilever thickness. This effect causes significant bending in coated 
cantilevers because of the difference in thermal expansion coefficient of the bulk and the coating.

The first part of the Appendix is devoted to derive the temperature profile along the cantilever caused by the local periodic heating because of absorption of the optical energy. In the second part, we evaluate the photothermal-induced force distribution $f_{\text {phot }}$.

The time-dependent temperature distribution $T(x, t)$ induced by the modulation of laser intensity can be described by the one-dimensional heat diffusion equation, ${ }^{30}$

$$
\frac{\partial T(x, t)}{\partial t}=K \frac{\partial^{2} T(x, t)}{\partial x^{2}}-\beta\left(T(x, t)-T_{\mathrm{amb}}\right)
$$

where $T_{a m b}$ is the temperature of the cantilever surrounding fluid and $K$ and $\beta$ are defined as

$$
\begin{gathered}
K=\frac{\kappa_{\text {eff }}}{c_{p_{\text {eff }} \rho_{\text {eff }}}}, \\
\beta=\frac{h_{\text {eff }} 2(W+D)}{c_{p_{\text {eff }} \rho_{\text {eff }} W D}} .
\end{gathered}
$$

Here, $k_{\text {eff }}, \rho_{\text {eff }}, c_{p_{\text {eff }}}$, and $h_{\text {eff }}$ indicate, respectively, the effective thermal conductivity, density, specific heat capacity, and heat-transfer coefficient of the cantilever, defined by the following equations:

$$
\begin{aligned}
\kappa_{\mathrm{eff}} & =\frac{\kappa_{1} D_{1}+\kappa_{2} D_{2}}{D_{1}+D_{2}}, \\
\rho_{\mathrm{eff}} & =\frac{\rho_{1} D_{1}+\rho_{2} D_{2}}{D_{1}+D_{2}}, \\
c_{p_{\mathrm{eff}}} & =\frac{c_{p_{1}} D_{1}+c_{p_{2}} D_{2}}{D_{1}+D_{2}}, \\
h_{\mathrm{eff}} & =\frac{h_{1} D_{1}+h_{2} D_{2}}{D_{1}+D_{2}},
\end{aligned}
$$

where the subscripts 1 and 2 denote, respectively, the coating film and the substrate material.

Assuming that the temperature distribution oscillates at the same frequency $\nu$ of the laser-intensity modulation yields,

$$
T(x, t)=T_{\mathrm{amb}}+\hat{T}(x) \exp (i 2 \pi \nu t),
$$

and, by substituting Eq. (A2) in (A1), we obtain

$$
\frac{\partial^{2} \hat{T}(x)}{\partial x^{2}}-\frac{\beta+i 2 \pi \nu}{K} \hat{T}(x)=0,
$$

where $\hat{T}(x)$ denotes the (complex) temperature variation with respect to the surrounding fluid. Moreover, imposing that the heat conducted outward from the cantilever-free end $x=L$ is equal to the one dissipated from the surface by free convection:

$$
\left.\frac{\partial \hat{T}(x)}{\partial x}\right|_{x=L}=-\left.\frac{h_{\mathrm{eff}}}{\kappa_{\mathrm{eff}}} \hat{T}(x)\right|_{x=L},
$$

and that the temperature at the focusing laser position $x=x_{0}$ is $\hat{T}_{0}$ :

$$
\left.\hat{T}(x)\right|_{x=x_{0}}=\hat{T}_{0},
$$

the solution of the Eq. (A3) can be written as:

$$
\begin{aligned}
\hat{T}(x)= & \hat{T}_{0} \exp \left[-f(\nu)\left|x-x_{0}\right|\right] \\
& +r \hat{T}_{0} \exp \left[f(\nu)\left(x+x_{0}-2 L\right)\right],
\end{aligned}
$$

where

$$
f(\nu)=\sqrt{\frac{\beta+i 2 \pi \nu}{K}} .
$$

The temperature distribution $\hat{T}(x)$ thus behaves as a damped oscillation, in which the temperature exponentially decays with the distance from the excitation position $x_{0}$ with a complex decay constant given by $f(\nu)$.

The second term of the solution (A6) represents the reflected thermal wave at the cantilever-free end, whose reflectivity coefficient $r$ can be obtained by exploiting the boundary condition (A4):

$$
r=\frac{-h_{\mathrm{eff}}+f(\nu) k_{\mathrm{eff}}}{h_{\mathrm{eff}}+f(\nu) k_{\mathrm{eff}}} .
$$

The quantity $\hat{T}_{0}$ represents the amplitude of the thermal wave and can be deduced by imposing a heat balance at $x=x_{0}$. In fact, the optical power absorbed by the cantilever must be equal to the heat loss. Here, three mechanisms of heat loss are relevant: heat loss by conduction, heat absorption by the cantilever material, and heat loss across the surface. The power lost by conduction is

$$
P_{1}=W D \kappa_{\mathrm{eff}}\left[\left.\frac{\partial T(x, t)}{\partial x}\right|_{x=x_{0}^{-}}-\left.\frac{\partial T(x, t)}{\partial x}\right|_{x=x_{0}^{+}}\right],
$$

whereas the heat absorbed per unit time can be written as

$$
P_{2}=\left.W D c_{p_{\text {eff }} \rho_{\text {eff }}} \Delta x \frac{\partial T(x, t)}{\partial t}\right|_{x=x_{0}}
$$

where $\Delta x$ accounts for the beam spot size of the modulated laser on the cantilever. Finally, the power loss across the surface is

$$
P_{3}=2 W h_{\mathrm{eff}} \Delta x\left(\left.T(x, t)\right|_{x=x_{0}}-T_{\mathrm{amb}}\right) .
$$

The absorbed optical laser power, $P_{0}=\hat{P}_{0} \exp (i 2 \pi \nu t)$, must be equal to the total energy loss per unit time

$$
P_{0}=P_{1}+P_{2}+P_{3}
$$

and imposing this balance provides an analytical expression for the thermal wave amplitude $\hat{T}_{0}$,

$$
\begin{gathered}
\hat{T}_{0}=\frac{\hat{P}_{0}}{2 W(a+b)}, \\
a=D f(\nu) k_{\mathrm{eff}}+h_{\mathrm{eff}} \Delta x+i c_{p_{\mathrm{eff}}} D \pi \Delta x \nu \rho_{\mathrm{eff}}, \\
b=r \Delta x \exp \left[f(\nu)\left(2 x_{0}-2 L\right)\right]\left(h_{\mathrm{eff}}+i c_{p_{\mathrm{eff}}} D \pi \nu \rho_{\mathrm{eff}}\right) .
\end{gathered}
$$

As we can see from these calculations, the complex quantities $r$ and $\hat{T}_{0}$ are implicitly frequency dependent. 
Let us now turn the focus to the evaluation of the photothermal-induced force distribution. The heat generated by the laser beam produces a differential longitudinal stress across the cantilever thickness because of the difference in the thermal expansion coefficients of the cantilever bulk and coating materials. The longitudinal stresses induced on the coating and on the substrate material are, respectively,

$$
\begin{array}{ll}
\sigma_{1}=\alpha_{1} E_{1} T(x, t) & z>0 \\
\sigma_{2}=\alpha_{2} E_{2} T(x, t) & z<0
\end{array},
$$

where $z=0$ identifies the interface between the coating and the substrate, $\alpha$ is the thermal expansion coefficient, and $E$ indicates the Young modulus. The differential longitudinal stress produces a bending moment given by

$$
\begin{gathered}
M_{\text {phot }}(x, t)=c_{\text {phot }} T(x, t), \\
c_{\text {phot }}=\frac{W}{2}\left(D_{1} E_{1} \alpha_{1}\left(D_{1}-2 z_{0}\right)-D_{2} E_{2} \alpha_{2}\left(D_{2}+2 z_{0}\right)\right),
\end{gathered}
$$

where $z_{0}$ is the neutral bending axis that is defined as ${ }^{31}$

$$
z_{0}=\frac{1}{2} \frac{E_{1} D_{1}^{2}+E_{2} D_{2}^{2}}{E_{1} D_{1}-E_{2} D_{2}} .
$$

Finally, the photothermal-induced bending moment allows to evaluate the force distribution:

$$
f_{\text {phot }}(x, t)=-\frac{\partial^{2} M_{\text {phot }}(x, t)}{\partial x^{2}} .
$$

Once the expression for the phothermal-induced force distribution is known, one can then obtain a prediction on the frequency response of the cantilever by simply integrating this distribution along each natural vibrational mode of the cantilever beam. ${ }^{20}$

${ }^{1}$ G. Binnig, C. F. Quate, and C. Gerber, Phys. Rev. Lett. 56, 930 (1986).

${ }^{2}$ N. Ookubo and S. Yumoto, Appl. Phys. Lett. 74, 2149 (1999).

${ }^{3}$ P. D. Ashby, Appl. Phys. Lett. 91, 254102 (2007).

${ }^{4}$ D. C. Hurley, K. Shen, N. M. Jennett, and J. A. Turner, J. Appl. Phys. 94, 2347 (2003).

${ }^{5}$ A. Vinckier and G. Semenza, FEBS Lett. 430, 12 (1998).
${ }^{6}$ T. R. Rodríguez and R. García, Appl. Phys. Lett. 84, 449 (2004).

${ }^{7}$ R. García and R. Perez, Surf. Sci. Rep. 47, 197 (2002).

${ }^{8}$ A. Passian and G. Muralidharan, J.Appl. Phys. 91, 4693 (2002).

${ }^{9}$ C.-W. Yang, I.-S. Hwang, Y. F. Chen, C. S. Chang, and D. P. Tsai, Nanotechnology 18, 084009 (2007).

${ }^{10}$ H. Sekiguchi, T. Okajima, H. Arakawa, S. Maeda, A. Takashima, and A. Ikai, Appl. Surf. Sci. 210, 61 (2003).

${ }^{11}$ T. R. Albrecht, P. Grütter, D. Horne, and D. Rugar, J. Appl. Phys. 69, 668 (1991).

${ }^{12}$ A. Schirmeisen, H. Hölscher, B. Anczykowski, D. Weiner, M. Schäfer, and H. Fuchs, Nanotechnology 16, S13 (2005).

${ }^{13}$ M. Basso, P. Paoletti, B. Tiribilli, and M. Vassalli, Nanotechnology 19, 475501 (2008).

${ }^{14}$ M. Basso, P. Paoletti, B. Tiribilli, and M. Vassalli, IEEE Trans. Nanotechnol. 10, 560 (2011).

${ }^{15}$ X. Xu and A. Raman, J. Appl. Phys. 102, 034303 (2007).

${ }^{16}$ J. Kokavecz and A. Mechler, Appl. Phys. Lett. 91, 023113 (2007).

${ }^{17}$ M. Allegrini, C. Ascoli, P. Baschieri, F. Dinelli, C. Frediani, A. Lio, and T. Mariani, Ultramicroscopy 42-44, 371 (1992).

${ }^{18}$ O. Marti, A. Ruf, M. Hipp, H. Bielefeldt, J. Colchero, and J. Mlynek, Ultramicroscopy 42-44, 345 (1992).

${ }^{19}$ S. Nishida, D. Kobayashi, H. Kawakatsu, and Y. Nishimori, J. Vac. Sci. Technol. B 27, 964 (2009).

${ }^{20}$ V. Pini, B. Tiribilli, C. M. C. Gambi, and M. Vassalli, Phys. Rev.B 81, 054302 (2010).

${ }^{21}$ A. Wig, A. Passian, E. Arakawa, T. L. Ferrell, and T. Thundat, J. Appl.Phys. 95, 1162 (2004)

${ }^{22}$ N. Umeda, S. Ishizaki, and H. Uwai, J. Vac. Sci. Technol. B 9, 1318 (1991).

${ }^{23}$ H. Yamashita, N. Kodera, A. Miyagi, T. Uchihashi, D. Yamamoto, and T. Ando, Rev. Sci. Instrum. 78, 083702 (2007).

${ }^{24}$ G. C. Ratcliff, D. A. Erie, and R. Superfine, Appl. Phys. Lett. 72, 1911 (1998).

${ }^{25}$ A. Wig, E. Arakawa, A. Passian, T. Ferrell, and T. Thundat, Sens. Actuators B 114, 206 (2006).

${ }^{26}$ D. Ramos, J. Mertens, M. Calleja, and J. Tamayo, Appl. Phys. Lett. 92, 173108 (2008).

${ }^{27}$ M. Basso, R. Bucher, M. Romagnoli, and M. Vassalli, "Real-Time Control with Linux: A Web Services Approach," in Decision and Control, 2005 and 2005 European Control Conference. CDC-ECC'05. 44th IEEE Conference on (IEEE, New York, 2005), pp. 2733-2738.

${ }^{28}$ D. Materassi, P. Baschieri, B. Tiribilli, G. Zuccheri, and B. Samori, Rev. Sci. Instrum. 80, 084301 (2009).

${ }^{29}$ Using cantilevers harder than $1 \mathrm{~N} / \mathrm{m}$ is actually not suitable for AO in liquid because of technological limitations of the setup, and thus we restricted the analysis to softer cantilevers.

${ }^{30}$ H. S. Carslaw and J. C. Jaeger, Conduction of Heat in Solids (Oxford University Press, New York, 1959).

${ }^{31}$ C. H. Hsueh, Thin Solid Films 418, 182 (2002). 